\title{
379.
}

\section{NOTICES OF COMMUNICATIONS TO THE BRITISH ASSOCIATION FOR THE ADVANCEMENT OF SCIENCE.}

[From the Reports of the British Association for the Advancement of Science, 1854 to 1860, Notices and Abstracts of miscellaneous Communications to the Sections.]

1. On the Solution of Cubic and Biquadratic Equations. Report, 1854, p. 1.

2. On the Porism of the In-and-circumscribed Triangle. Report, 1855, p. 1.

THE porism of the in-and-circumscribed triangle in its most general form relates to a triangle the angles of which lie in fixed curves, and the sides of which touch fixed curves, but at present I consider only the case in which the angles lie in one and the same fixed curve which for greater simplicity I consider to be a conic. We have therefore a triangle $A B C$ the angles of which lie in a fixed conic $\subseteq$ and the sides of which touch the fixed curves $\mathfrak{A}, \mathfrak{B}$, $\mathfrak{\complement}$. And if we consider the conic $\subseteq$ and the curves $\mathfrak{A}, \mathfrak{B}$ as given, the curve $\mathfrak{\complement}$ will be the envelope of the side $A B$ of the triangle. Suppose that the curves $\mathfrak{A}, \mathfrak{B}$ are of the classes $m, n$ respectively; there is no difficulty in showing that the curve $(5$ is of the class $2 \mathrm{mn}$. But the curve (5 has in general double tangents forming two distinct groups, the first group arising from the quadrilaterals inscribed in the conic $\mathbb{S}$ and such that two opposite sides touch the curve $\mathfrak{A}$, and the other two opposite sides the curve $\mathfrak{B}$; the second group arising from quadrilaterals such that two adjacent sides touch the curve $\mathfrak{A}$ and the other two adjacent sides touch the curve $\mathfrak{B}$. The number of double tangents of the first group is $=m n(m n-1)$, and the number of double tangents of the second group is $=m n(m n-m-n+1)$; the number of double tangents of the two groups is therefore $=m n(2 m n-m-n)$. The curve $(5$ has not in general any inflexions, hence, being of the class $2 m n$ with $m n(2 m n-m-n)$ double tangents, it will be of the order $2 m n(m+n-1)$. 
When the curves $\mathfrak{A}$ and $\mathfrak{B}$ are conics, the curve $\mathfrak{B}$ is therefore of the class 8 , with 16 double tangents but no inflexions, consequently of the order 24 . But there are two remarkable cases in which the order is further diminished.

First when each of the conics $\mathfrak{N}, \mathfrak{B}$ has double contact with the conic $\mathfrak{S}$. The four points of contact give rise to 8 new double tangents or there are in all 24 double tangents, the curve 5 is therefore of the degree 8 : and being of the class 8 with 24 double tangents, it must of necessity break up into 4 curves each of the class 2, i.e. into 4 conics. Each of these has double contact with the conic $\subseteq$, or attending to only one of the four conics we have the well-known theorem which I call the porism (homographic) of the in-and-circumscribed triangle, viz. "there are an infinity of triangles inscribed each in a conic, and such that the sides touch conics having each of them double contact with the circumscribed conic."

Secondly, the conics $\mathfrak{A}$ and $\mathfrak{B}$ may intersect the conic $\mathfrak{S}$ in the same four points. Here every tangent of the curve $\sqrt{5}$ is in fact a double tangent belonging to the first-mentioned group, the curve 5 in fact consists of two coincident curves: each of them is therefore of the class 4. But this curve of the class 4 has itself four double tangents arising from the common points of intersection of the conics $\mathfrak{A}, \mathfrak{B}$ with the conic $\mathfrak{S}$; it must therefore break up into two curves each of the class 2 , i.e. into two conics: each of these intersects the conic $\mathfrak{S}$ in the same four points in which it is intersected by the conics $\mathfrak{A}, \mathfrak{B}$. Attending only to one of the two conics we have the other well-known theorem which I call the porism (allographic) of the in-and-circumscribed triangle, viz. "there exist an infinity of triangles inscribed in a conic, and such that the sides touch conics, each of them meeting the circumscribed conic in the same four points."

\section{On the Notion of Distance in Analytical Geometry. Report, 1858, p. 3.}

THE author remarks that the principles of-Modern Geometry show that any metrical property whatever is really based upon a purely descriptive property, and that these principles contain in fact a theory of distance-but that such theory has not been disengaged from its applications and stated in a distinct and explicit form. The paper contains an account of the theory in question, viz. it is shown that in any system of geometry of two dimensions the notion of distance can be arrived at from descriptive principles by means of a conic called the Absolute, and which in ordinary geometry degenerates into a pair of points.

\section{On Curves of the Fourth Order having Three Double Points. Report, 1860, p. 4.}

THE paper is a short notice only of researches which the Author is engaged in with reference to curves of the fourth order having three double points. A curve of the kind in question is derived from a conic by the well-known transformation of substituting for the original trilinear coordinates their reciprocals: and the species of the curve of the fourth order depends on the position of the conic with respect to the fundamental triangle. 
5. On Curves of the Third Order. Report, 1861, p. 2.

A CURve of the third order or cubic curve is a section of a cubic cone and such cone is intersected by a concentric sphere in a spherical cubic. It is an obvious consequence of a theorem of Sir Isaac Newton's that there are five principal kinds of cubic cones, or what is the same thing five principal kinds of spherical cubicsbut the nature of these five kinds of spherical cubics was first distinctly explained by Möbius. They may be designated the simplex, the complex, the crunodal, the acnodal and the cuspidal: where crunode, acnode, denote respectively the two species of double points (nodes), viz. the double point with two real branches, and the conjugate or isolated point. The foregoing results are known: the special object of the paper is to establish a subdivision of the simplex kind of spherical cubics. The simplex kind is a continuous reentering curve cutting a great circle, to fix the ideas say the equator, in three pairs of opposite points, which are the three real inflexions of the curve. The three great circles which are the tangents at the inflexions and the equator divide the entire surface of the sphere into fourteen regions whereof eight are trilateral and the remaining six are quadrilateral. The curve may be entirely in six out of the eight trilateral regions, and it is in this case said to be simplex trilateral; or it may lie entirely in the six quadrilateral regions, and it is in this case said to be simplex quadrilateral; and there is an intermediate form, the simplex neutral; viz. in this case the three great circles tangents at the inflexions meet in a pair of opposite points and there are in all only twelve regions all of them trilateral; the curve lies entirely in six of these regions.

6. On a Certain Curve of the Fourth Order. Report, 1862, p. 3.

THE curve in question is the locus of the centres of the conics which pass through three given points and touch a given line; if the equations of the sides of the triangle formed by the three points are $x=0, y=0, z=0$, these coordinates being such that $x+y+z=0$ is the equation of the line infinity, and if $\alpha x+\beta y+\gamma z=0$ be the equation of the given line, then (as is known) the equation of the curve is

$$
\sqrt{\alpha x(y+z-x)+\sqrt{ } \beta y}(z+x-y)+\sqrt{\gamma z}(x+y-z)=0 .
$$

The special object of the communication was to exhibit the form of the curve in the case where the line cuts the triangle, and to point out the correspondence of the positions of the centre upon the curve, and the point of contact on the given line. 


\section{On the Representation of a Curve in Space by means of a Cone and Monoid} Surface. Report, 1862, p. 3.

THE author gave a short account of his researches recently published in the Comptes Rendus. The difficulty as to the representation of a curve in space is, that such a curve is not in general the complete intersection of two surfaces; any two surfaces passing through the curve intersect not only in the curve itself, but in a certain companion curve, which cannot be got rid of; this companion curve is in the proposed mode of representation reduced to the simplest form, viz. that of a system of lines passing through one and the same point. The two surfaces employed for the representation of a curve of the $n$th order are, a cone of the $n$th order having for its vertex an arbitrary point (say the point $x=0, y=0, z=0$ ), and a monoid surface with the same vertex, viz. a surface the equation whereof is of the form $Q w-P=0$, $P$ and $Q$ being homogeneous functions of $(x, y, z)$ of the degrees $p$ and $p-1$ respectively (where $p$ is at most $=n-1$ ). The monoid surface contains upon it $p(p-1)$ lines given by the equations $(P=0, Q=0)$; and, the cone passing through $n(p-1)$ of these lines (if, as above supposed, $p \ngtr n-1$, this implies that some of these lines are multiple lines of the cone), the monoid surface will besides intersect the cone in a curve of the $n$th order.

8. On a Formula of $M$. Chasles relating to the Contact of Conics. Report, 1864, p. 1.

THE author gave an account of the recent investigations of $\mathrm{M}$. Chasles in relation to the theory of conics, viz., M. Chasles has found that the properties of a system of conics, containing one arbitrary parameter, depend upon two quantities called by him the characteristics of the system; these are, $\mu$, the number of conics of the system which pass through a given point, and, $\nu$, the number of conics of the system which touch a given line; or, say, $\mu$ is the parametric order, $\nu$ the parametric class, of the system. And he exhibited a transformation obtained by him of a formula of M. Chasles for the number of conics which touch five given curves, viz., if $(M, m)$ $(N, n)(P, p)(Q, q)(R, r)$ be the orders and classes of the five given curves respectively, then the number of curves is

$$
=(1,2,4,4,2,1)(M, m)(N, n)(P, p)(Q, q)(R, r)
$$

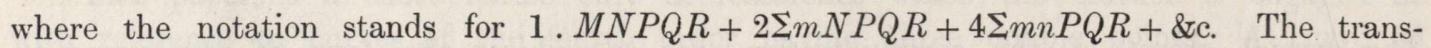
formed formula in question was communicated by the author to M. Chasles, and had appeared in the Comptes Rendus; but it is, in fact, included in a very beautiful and general theorem given in the same Number by M. Chasles himself. 
9. On the Problem of the In-and-circumscribed Triangle. Report, 1864, p. 1.

THE general problem of the in-and-circumscribed triangle may be thus stated, viz., to find a triangle the angles whereof severally lie in, and the sides severally touch, a given curve or curves; and we may, in the first instance, inquire as to the number of such triangles. The first and easiest case is when the curves are all distinct; here, if the angles lie in curves of the orders $m, n, p$, respectively, and the sides touch curves of the classes $Q, R, S$, respectively, then the number of triangles is $=2 m n p Q R S$. The number may be obtained for some other cases; but the author has not yet considered the final and most difficult case, viz. that in which the angles severally lie in, and the sides severally touch, one and the same given curve.

The foregoing notices relate to verbal communications upon questions with which I was at the time occupied and which are for the most part more fully discussed in papers printed elsewhere. I remark upon them as follows:

1. I have no remembrance as to this; I think no paper printed or written.

2. See 17 .

3. See 158 .

4. No paper printed. The intention was to consider the different forms of trinodal quartic curves, in particular those with real nodes, as obtained from the inversion of a conic according to the different relations of the conic to the fundamental triangle. Thus according as the conic cuts in two real points, touches, or cuts in two imaginary points, a side of the triangle, the tangents at the corresponding node are real, coincident, or imaginary; viz. the node is a crunode, cusp, or acnode. And in the case of real intersections there is a further distinction according as the intersections lie each or either of them on the side itself, or on the side produced in one or other of the two directions. By considering the different relations of the conic to the fundamental triangle we thus obtain the different forms of the trinodal quartic.

5. See 351.

6. I think no paper printed or written.

7. See 302 and 305 .

8. See 306 .

9. The question is considered in a memoir On the Problem of the In-and-circumscribed Triangle, Phil. Trans. t. CLXI. (for 1871), pp. 369-412. 